\title{
Numerical and Theoretical Investigation to Estimate Darcy Friction Factor in Water Network Problem Based on Modified Chun-Hui He's Algorithm and Applications
}

\author{
Waseem Asghar Khan $\left(\mathbb{D},{ }^{1}\right.$ Muhammad Arif ${ }^{D},{ }^{2}$ Mogtaba Mohammed, ${ }^{1,3}$ Umer Farooq ${ }^{D},{ }^{4}$ \\ Fozia Bashir Farooq, ${ }^{5}$ Murtada K. Elbashir $\left(\mathbb{0},{ }^{6}\right.$ Jamshaid Ul Rahman, \\ and Ziyad Ali AlHussain ${ }^{1}$ \\ ${ }^{1}$ Department of Mathematics, Faculty of Sciences AlZulfi, Majmaah University, Majmaah 11952, Saudi Arabia \\ ${ }^{2}$ Department of Mathematics, Abdul Wali Khan University Mardan, Mardan 23200, Pakistan \\ ${ }^{3}$ Faculty of Mathematical and Computer Sciences, University of Gezira, Wad Madani 11123, Sudan \\ ${ }^{4}$ Department of Mathematics, COMSATS University Islamabad, Park Road Chak Shahzad, Islamabad 44000, Pakistan \\ ${ }^{5}$ Department of Mathematics, Imam Muhammad Ibn Saud Islamic University, Riyadh, Saudi Arabia \\ ${ }^{6}$ College of Computer and Information Sciences, Jouf University, Sakaka 72441, Saudi Arabia \\ ${ }^{7}$ Abdus Salam School of Mathematical Sciences, GCU, Lahore 54000, Pakistan \\ Correspondence should be addressed to Waseem Asghar Khan; wa.khan@mu.edu.sa
}

Received 21 September 2021; Accepted 20 January 2022; Published 15 February 2022

Academic Editor: Alessandro Rasulo

Copyright ( 2022 Waseem Asghar Khan et al. This is an open access article distributed under the Creative Commons Attribution License, which permits unrestricted use, distribution, and reproduction in any medium, provided the original work is properly cited.

\begin{abstract}
In this work, we applied the modified Chun-Hui He's algorithm to evaluate for estimation of flow friction factor $f$ for value of friction factor by using Colebrook-White relation. The speedy, precise, and consistent evaluation of flow friction factor $f$ are essential for evaluation of pressure dips and streams in complex network prototypes at distinct values of diameters of pipes. Friction factor estimated outcomes are applied in everyday engineering routine. Numerous computational systems tested for distinguishing of water pipe networks resolution, such as Hardy Cross method (HCM), Newton method (NM), and modified Newton method (MNM), are presented. As a novelty, a modified Newton method tabulated data, graphical results, and comparisons that are presented with different numerical schemes.
\end{abstract}

\section{Introduction}

The investigation of a few issues in many fields such as computational physics, computational biology, engineering, environmental sciences, chemistry, and economics in order to resolve real-life nonlinear models with constrained domain. The Newton technique and its variations are effective in solving nonlinear models that occur in real-world problems with reasonable stopping conditions $[1,2]$. The flow variation to all loops is immediately removed in this manner, resulting in a high-level merger. This technique nevertheless requires suitable essential assumptions for tributary levels that sustain progression circumstances and are adjacent to the specific stream $[3,4]$.

The stream systems are energetic, and complex frameworks involve gigantic ventures by private and government sectors. These sectors need sufficient management to control professionally to accomplish goals of their system [5-11]. Unfortunately, now a days, the management of water resource systems is challenging and problematic due to the rapidly increasing customer requirements. This situation is difficult and challenging for conventional methodologies to manage these circumstances [12-14]. Recently, computational methodologies have been tried to tackle these complex circumstances $[11,12]$. To explore hydraulic movement in 
complicated network systems and to satisfy the energy and continuity equations, these quantitative approaches use the Hardy Cross method. Various research laboratories have recently used various techniques to solve these limitations, such as sluggish convergence and recurring dissatisfaction with outcomes, and have failed to meet consumer demand criteria. The failure of others' attempts to eliminate all problems sparked the breakthrough. Furthermore, the Hardy Cross method must be modified due to the nonlinear nature of tube systems $[5,15,16]$.

In electrical systems, the relationship between voltage and current with regular resistors is governed by Ohm's law with diodes where resistances depending on current and voltage are nonlinear electrical circuits containing nonlinear components and solving second-order coupled nonlinear Schrödinger equations by using various numerical approaches [3, 4]. HCM is followed by other methods that adopted the Newton-Raphson method, one of the faster effective procedures with higher convergence [17-19]. Toldini and Pilati [20] proposed a global gradient method that is created as a variation to the NM. Such methodologies are usually applied to resolve a system of nonlinear algebraic equations that communicate the behaviors of the hydraulic structures [21-23]. To solve nonlinear problems, numerical approaches are a reasonable alternative. The well-known numerical approaches are visible in [3].

In this study, we believe in a new estimated scheme that is a variation of the standard NM, and we evaluated its efficacy alongside the NM and HCM. At Re $\geq 4000$, the modified Chun-Hui He's algorithm [24] was used to calculate the friction factor for given pipe diameters in turbulent flow in a confined region, where Re is called Reynolds number and $\varepsilon=0.05$ is roughness height $[25,26]$. At the next level, we used fraction factor values to obtain the water pressure function for various lengths, diameters, and water flow rates in each pipe. All computational data are first incorporated into an Excel sheet, and then, Mathematica code is used to describe the consequences of network systems using Excel data [6].

\section{Structure Topology of the Hydraulic System}

The first step in characterizing a hydraulic problem is to create a network composition that shows pipe connections in terms of diameter, length, and nodes. Water and utilization levels from suppliers should be allocated to intersection points. Instead of using availability to track pipes, metrics are assigned to each tube and the system's closed loop, as shown in Figure 1. The next step demonstrates the pipes system for preliminary supply of stream is used for utilization in every intersection point and should obey Kirchhoff's law [27]. The overall water entering at an intersection point is approximately the same of that leaves that enter intersection point of the network. The similar preservation law is to satisfy the entire system.

\section{Topology of the Hydraulic Model}

A scientific explanation of the model can be developed once a complex system configuration is created, along with its loop numbers, pipeline, supply, and resource data. According to the mineralogy theorem of Euler, $\mathrm{M}$ nodes (intersection points) and $\mathrm{N}$ branches make up the system. In above network problem, we have $N=13$ and $M=10$ and $M-1$ autonomous intersection points, i.e., 9 points and other one point is known as referent point and $N-M+1=$ $13-10+1=4$ autonomous loops. In over problem, point $\mathbf{J}$ is called referent node.

The Darcy-Weisbach equation and Colebrook-White relation for the Darcy friction factor $(f)$ can be used to analyze this pipe network $[8,28]$.

$$
W_{w} \approx \Delta w=w_{1}-w_{2}=\frac{8 \mathrm{fL} \mathbf{q}^{2}}{g D^{5} \pi^{2}},
$$

where $W_{w}$ is the water pressure function. $L=\left(l_{i}(m)\right), D=$ $\left(d_{i}(m)\right)$ and $\mathbf{q}=\left(q_{i}\left(m^{3} / s\right)\right)$ for $i=1,2, \ldots, 13$ are the pipes length, diameter, and flow vectors, respectively, and $\mathbf{g}$ is the gravity $\left(\mathrm{m} / \mathrm{s}^{2}\right)$.

Taking the first derivative of equation (1) and $\mathbf{q}$ assumed as a variable herewith, we have

$$
W_{w}^{\prime} \approx \frac{\partial W_{w}(\mathbf{q})}{\partial \mathbf{q}}=\frac{16 \mathrm{fL} \mathbf{q}}{g D^{5} \pi^{2}} .
$$

Darcy friction factor $(f)$ can be described as

$$
\frac{1}{\sqrt{f}}=-2 \log _{10}\left[\frac{\varepsilon}{3.71(\text { diameter })}+\frac{2.51}{\operatorname{Re} \cdot \sqrt{f}}\right] .
$$

Colebrook-White (3) is applicable only at turbulent flow in restricted domain at $\mathrm{Re} \geq 4000$, where Re is called Reynolds number and $\varepsilon=0.05$ is roughness height $[25,26,28]$.

3.1. Modified Chun-Hui He's Algorithm for Friction Factor (f). The quick, precise, and dependable evaluation of friction factor $(f)$ in (3) are essential for estimation of pressure falls in complex network models $[6,29]$. So, we utilize the modified Chun-Hui He's algorithm [24] to solve the approximate value of friction factor $f$ from equation (3), and we found that the modified Chun-Hui He's algorithm is upto-date, and an efficient algorithm for solving nonlinear equations exists in real-life applications.

(3) can be redeveloped as follows:

$F\left(f, \frac{\varepsilon}{D}, \operatorname{Re}\right)=\frac{1}{\sqrt{f}}+2 \log _{10}\left[\frac{\varepsilon}{3.71(\text { Diameter })}+\frac{2.51}{\operatorname{Re} \cdot \sqrt{f}}\right]=0$.

The friction factor $f$ is used as a variable in this case, and we carefully select preliminary estimates to begin the numerical method in the given restricted domain. 


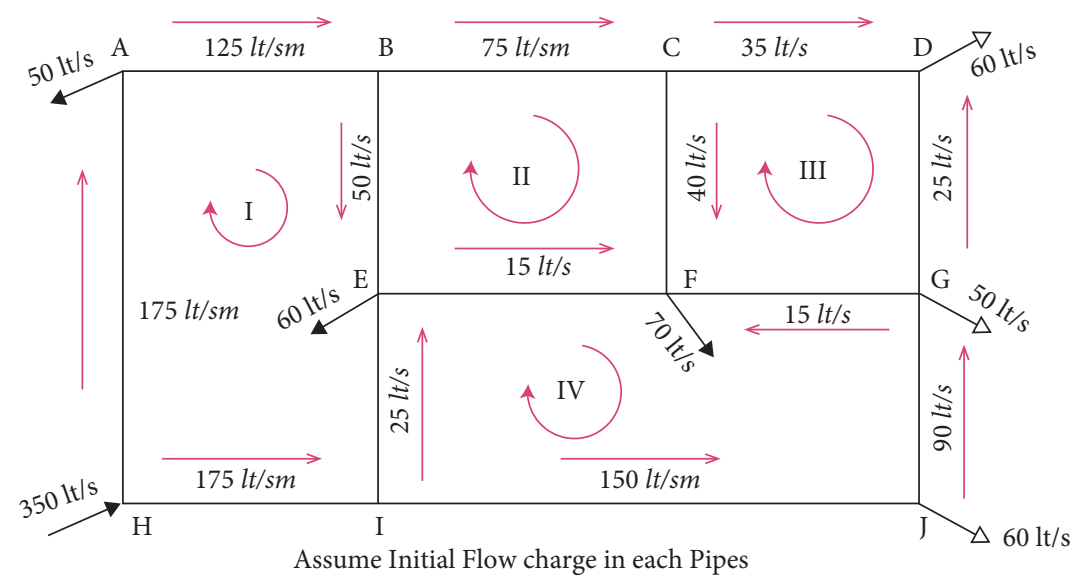

Figure 1: The problem with the water supply network prompted by Kirchhoff's law [3].

\subsubsection{Modified Chun-Hui He’s Algorithm [24]}

Step 1. Necessary condition.

$$
F\left(x_{0}\right) F\left(x_{1}\right)<0,
$$

where $x_{0}$ and $x_{1}$ are the starting assumptions.

Step 2. Ancient Chinese algorithm computes

$$
x_{2}=x_{0}-\frac{F\left(x_{0}\right)}{R\left(x_{0}, x_{1}\right)},
$$

where $R\left(x_{0}, x_{1}\right)=\left(F\left(x_{0}\right)-F\left(x_{1}\right)\right) /\left(x_{0}-x_{1}\right)$.

Step 3. Corrector step:

$$
x_{3}=x_{2}-\frac{F\left(x_{2}\right)}{F^{\prime}\left(x_{2}\right)} .
$$

Step 4. Assumptions:

$$
\begin{aligned}
& x_{0}=x_{3}-\delta, \\
& x_{1}=x_{3}+\delta, \\
& \delta=-\frac{F\left(x_{3}\right)}{F^{\prime}\left(x_{3}\right)} .
\end{aligned}
$$

Step 5. If we not get required accuracy, then go back to Step 2.

Table 1 provides the estimated fraction factor $f_{n}$ flow numbers using the modified Chun-Hui He's Algorithm [24] for various pipe diameters (m).
3.2. Loops Model in Water Network Topology. The system of nonlinear equations along each loop in this section was simulated.

Loop I: $W_{I}$,

$\Delta w_{1}+\Delta w_{9}-\Delta w_{13}-\Delta w_{7}+\Delta w_{8}$

$$
\begin{aligned}
= & \frac{8}{g \pi^{2}}\left(\frac{f_{1} l_{1} q_{1}^{2}}{d_{1}^{5}}+\frac{f_{9} l_{9} q_{9}^{2}}{d_{9}^{5}}-\frac{f_{13} l_{13} q_{13}^{2}}{d_{13}^{5}}-\frac{f_{7} l_{7} q_{7}^{2}}{d_{7}^{5}}+\frac{f_{8} l_{8} q_{8}^{2}}{d_{8}^{5}}\right), \\
= & 222.5804287 q_{1}^{2}+536.2707478 q_{9}^{2}-91.8100660 q_{13}^{2} \\
& -1708.9539397 q_{7}^{2}+3987.5591927 q_{8}^{2} .
\end{aligned}
$$

Loop II: $W_{I I}$,

$$
\begin{aligned}
\Delta w_{2} & +\Delta w_{10}-\Delta w_{11}-\Delta w_{9} \\
= & \frac{8}{g \pi^{2}}\left(\frac{f_{2} l_{2} q_{2}^{2}}{d_{2}^{5}}+\frac{f_{10} l_{10} q_{10}^{2}}{d_{10}^{5}}-\frac{f_{11} l_{11} q_{11}^{2}}{d_{11}^{5}}-\frac{f_{9} l_{9} q_{9}^{2}}{d_{9}^{5}}\right), \\
= & 278.2255359 q_{2}^{2}+53.6270748 q_{10}^{2}-53.6270748 q_{11}^{2} \\
& -536.2707478 q_{9}^{2} .
\end{aligned}
$$

Loop III: $W_{I I I}$,

$$
\begin{aligned}
\Delta w_{3}-\Delta w_{4}+\Delta w_{12}-\Delta w_{10} \\
=\frac{8}{g \pi^{2}}\left(\frac{f_{3} l_{3} q_{3}^{2}}{d_{3}^{5}}-\frac{f_{4} l_{4} q_{4}^{2}}{d_{4}^{5}}+\frac{f_{12} l_{12} q_{12}^{2}}{d_{12}^{5}}-\frac{f_{10} l_{10} q_{10}^{2}}{d_{10}^{5}}\right), \\
=278.2255359 q_{3}^{2}-536.2707478 q_{4}^{2}+53.6270748 q_{12}^{2} \\
\quad-53.6270748 q_{10}^{2} .
\end{aligned}
$$


TABLE 1: The statistics values of flow, diameter, length, and constants value $\mathrm{K}$ displayed along with each value of the fraction factor $f$ of each pipe in the network (Figure 2).

\begin{tabular}{|c|c|c|c|c|c|c|c|}
\hline Pipes & No. & $f_{n}$ & Assume flow (lt/s) & Assume flow $\left(\mathrm{m}^{3} / \mathrm{s}\right)$ & Diameter (m) & Length $(\mathrm{m})$ & $K=8 \mathrm{fL} / \mathrm{gD}^{5} \mathrm{U}^{2}$ \\
\hline$A-B$ & 1 & 0.4470154801206153 & $q_{1}=125$ & $q_{1}=0.125$ & 0.457 & 120 & 222.5804287 \\
\hline B-C & 2 & 0.4470154801206153 & $q_{2}=75$ & $q_{2}=0.075$ & 0.457 & 150 & 278.2255359 \\
\hline C-D & 3 & 0.4470154801206153 & $q_{3}=35$ & $q_{3}=0.035$ & 0.457 & 150 & 278.2255359 \\
\hline$D-G$ & 4 & 0.4470232660486057 & $q_{4}=25$ & $q_{4}=0.025$ & 0.406 & 160 & 536.2707478 \\
\hline G-J & 5 & 0.4470154801206153 & $q_{5}=90$ & $q_{5}=0.090$ & 0.457 & 120 & 222.5804287 \\
\hline J-I & 6 & 0.4470000072957691 & $q_{6}=150$ & $q_{6}=0.150$ & 0.609 & 300 & 132.4056409 \\
\hline $\mathrm{I}-\mathrm{H}$ & 7 & 0.4470466694856617 & $q_{7}=175$ & $q_{7}=0175$ & 0.304 & 120 & 1708.9539397 \\
\hline $\mathrm{H}-\mathrm{A}$ & 8 & 0.4470466694856617 & $q_{8}=175$ & $q_{8}=0175$ & 0.304 & 280 & 3987.5591927 \\
\hline B-E & 9 & 0.4470232660486057 & $q_{9}=50$ & $q_{9}=0.050$ & 0.406 & 160 & 536.2707478 \\
\hline $\mathrm{C}-\mathrm{F}$ & 10 & 0.4470232660486057 & $q_{10}=40$ & $q_{10}=0.040$ & 0.406 & 16 & 53.6270748 \\
\hline E-F & 11 & 0.4470232660486057 & $q_{11}=15$ & $q_{11}=0.015$ & 0.406 & 16 & 53.6270748 \\
\hline F-G & 12 & 0.4470232660486057 & $q_{12}=15$ & $q_{12}=0.015$ & 0.406 & 16 & 53.6270748 \\
\hline E-I & 13 & 0.44703328769837464 & $q_{13}=25$ & $q_{13}=0.025$ & 0.355 & 14 & 91.8100660 \\
\hline
\end{tabular}

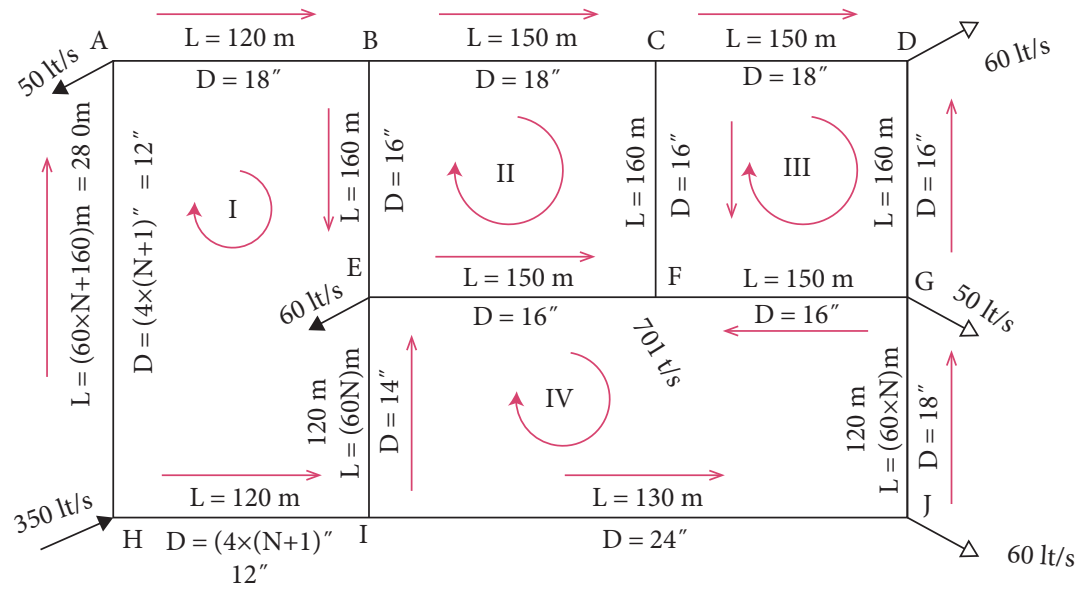

Figure 2: Water supply network problem [3].

Loop IV: $W_{I V}$,

$$
\begin{aligned}
& -\Delta w_{5}-\Delta w_{6}+\Delta w_{13}+\Delta w_{11}-\Delta w_{12} \\
& =\frac{8}{g \pi^{2}}\left(-\frac{f_{5} l_{5} q_{5}^{2}}{d_{5}^{5}}-\frac{f_{6} l_{6} q_{6}^{2}}{d_{6}^{5}}+\frac{f_{13} l_{13} q_{13}^{2}}{d_{13}^{5}}+\frac{f_{11} l_{11} q_{11}^{2}}{d_{11}^{5}}-\frac{f_{12} l_{12} q_{12}^{2}}{d_{12}^{5}}\right) . \\
& =-222.5804287 q_{5}^{2}-132.4056409 q_{6}^{2}+91.8100660 q_{13}^{2}+53.6270748 q_{11}^{2}-53.6270748 q_{12}^{2} .
\end{aligned}
$$

Above loop relations, $W_{I}, W_{I I}, W_{I I I}$, and $W_{I V}$ can be written in matrix form:

$$
\left[\begin{array}{ccccccccccccc}
1 & 0 & 0 & 0 & 0 & 0 & -1 & 1 & 1 & 0 & 0 & 0 & -1 \\
0 & 1 & 0 & 0 & 0 & 0 & 0 & 0 & -1 & 1 & -1 & 0 & 0 \\
0 & 0 & 1 & -1 & 0 & 0 & 0 & 0 & 0 & -1 & 0 & 1 & 0 \\
0 & 0 & 0 & 0 & -1 & -1 & 0 & 0 & 0 & 0 & 1 & -1 & 1
\end{array}\right] \times\left[\begin{array}{c}
\Delta w_{1} \\
\Delta w_{2} \\
\Delta w_{3} \\
\Delta w_{4} \\
\Delta w_{5} \\
\vdots \\
\Delta w_{13}
\end{array}\right]=0
$$


where $l_{1}, l_{2}, \ldots, l_{13}$ are the lengths of each pipe, $d_{1}, d_{2}, \ldots, d_{13}$ are the diameters, and water flow in each pipe, as shown in Figure 1, is $q_{1}, q_{2}, \ldots, q_{13}$, respectively. The positive sign showing flow direction is clockwise in left matrix of (14) and vice versa $[5,9,30]$.

3.3. Nodes Model Topology. In this section, we will simulate the system of linear equation along each node by using Kirchhoff's first law:

(1) Node A: $\quad-q_{1}+q_{8}-q_{A-\text { output }}=0, \quad$ where $q_{\text {A-output }}=50 \mathrm{lt} / \mathrm{s}$

(2) Node B: $\quad q_{1}-q_{2}-q_{9}-q_{B-\text { output }}=0$, where $q_{B-\text { output }}=0$

(3) Node C: $q_{2}-q_{3}-q_{10}-q_{C \text {-output }}=0$, where $q_{\mathrm{C} \text {-output }}=0$

(4) Node D: $\quad q_{3}+q_{4}-q_{D-\text { output }}=0, \quad$ where $q_{D-\text { output }}=60 \mathrm{lt} / \mathrm{s}$
(5) Node E: $q_{9}+q_{13}-q_{11}-q_{E-\text { output }}=0$, where $q_{E-\text { output }}=60 \mathrm{lt} / \mathrm{s}$

(6) Node F: $q_{11}+q_{10}+q_{12}-q_{F-\text { output }}=0$, where $q_{F-\text { output }}=70 \mathrm{lt} / \mathrm{s}$

(7) Node G: $\quad q_{5}-q_{4}-q_{12}-q_{G-\text { output }}=0$, where $q_{G-\text { output }}=50 \mathrm{lt} / \mathrm{s}$

(8) Node H: $-q_{7}-q_{8}+q_{H \text {-input }}-q_{H-\text { output }}=0$, where $q_{H-\text { output }}=0, q_{H-\text { input }}=350$

(9) Node I: $\quad q_{7}-q_{6}-q_{13}-q_{I-\text { output }}=0$, where $q_{I-\text { output }}=0$

(10) Node J: $\quad q_{6}-q_{5}-q_{J-\text { output }}=0 \quad$ where $q_{J \text {-output }}=60 \mathrm{lt} /$ sand referent.

Now, rewrite the above linear system of equations in matrix form [31]. The drawback of this matrix form is not a linear independent, that is why referent node would be omitted from the system of linear equation.

$$
\left[\begin{array}{cccccccccccc}
1 & 0 & 0 & 0 & 0 & 0 & 1 & 0 & 0 & 0 & 0 & 0 \\
1 & -1 & 0 & 0 & 0 & 0 & 0 & -1 & 0 & 0 & 0 & 0 \\
0 & 1 & -1 & 0 & 0 & 0 & 0 & 0 & -1 & 0 & 0 & 0 \\
0 & 0 & 1 & 1 & 0 & 0 & 0 & 0 & 0 & 0 & 0 & 0 \\
0 & 0 & 0 & 0 & 0 & 0 & 0 & 1 & 0 & -1 & 0 & 1 \\
0 & 0 & 0 & 0 & 0 & 0 & 0 & 0 & 1 & 1 & 1 & 0 \\
0 & 0 & -1 & 1 & 0 & 0 & 0 & 0 & 0 & 0 & -1 & 0 \\
0 & 0 & 0 & 0 & 0 & -1 & -1 & 0 & 0 & 0 & 0 & 0 \\
0 & 0 & 0 & 0 & -1 & 1 & 0 & 0 & 0 & 0 & 0 & -1
\end{array}\right]_{(9 \times 13)}
$$

Above node $\mathbf{J}$ count up as a referent, the left matrix in system (15) shows the rows corresponding to node $\mathbf{A}$, node B, ... node I, respectively.

3.4. Hardy Cross Method. The Hardy Cross method is applied to multiloop water network problems. Figures 3 and 4 show the complete results of the HCM. This method converges to the required results after $24^{\text {th }}$ iteration.

The primary stage in resolution the problem is to make a net map display lengths and diameters and networks nodes. Later, we compose the original stream supply all through a pipe network. The selection of original flows must fulfill Kirchhoff's law used in the system, and conservation law is also applicable for the entire network system $[3,13,14,27]$.

Outcomes of HCM in column 7 indicate that after $24^{\text {th }}$ iteration $-\mathbf{W}_{\mathbf{w}} /\left|\mathbf{W}_{\mathbf{w}}^{\prime}\right|$ (head loss) is nil by Darcy-Weisbach relation (1). The negative sign indicates anticlockwise water flows shown in Figure 1. Assign a sign with new flow of water. We will add in clockwise if $\Delta Q$ is +ve and vice versa. Similarly, we will subtract value if $\Delta Q$ is -ve and vice versa.

$$
\left[\begin{array}{c}
q_{1} \\
q_{2} \\
q_{3} \\
q_{4} \\
q_{5} \\
\vdots \\
q_{13}
\end{array}\right]_{(13 \times 1)}=\left[\begin{array}{c}
q_{A}-\text { output }=50 \\
q_{B}-\text { output }=0 \\
q_{C}-\text { output }=0 \\
q_{D}-\text { output }=60 \\
q_{E}-\text { output }=60 \\
q_{F}-\text { output }=70 \\
q_{G}-\text { output }=50 \\
q_{H-\text { output }}-q_{H-\text { input }}=-350 \\
q_{I}-\text { output }=0
\end{array}\right]_{(9 \times 1)}
$$

3.5. Newton Method. In this paragraph, hydrological problem is investigated by utilizing the NM having $2^{\text {nd }}$-order [32]. NM [23] or Newton-like procedure is not essential to take up an original estimate that fulfills the continuity law [33], as shown in Figure 1.

Table 2 provides the numerical results of correction flow of (1) by using conventional NM and change in two consecutive flows in each loop going to zero after $5^{\text {th }}$ iteration by selecting the random original flow in network problem. The effectiveness of NM is better than HCM.

3.6. MNM (Modified Newton Method). In this subsection, MNM achieves an optimum resolution of the nonlinear model simulated in Sections 3.2 and 3.3 for scrutinizing the hydrological network considering. Present procedure is cost-effective and takes a smaller amount of time to attain the results than the HCM and conventional NM.

Table 3 provides the numerical results of correction flow of (1) by using MNM and change in two consecutive flows in each loop going to zero after three iterations by selecting the random original flow in network problem. 


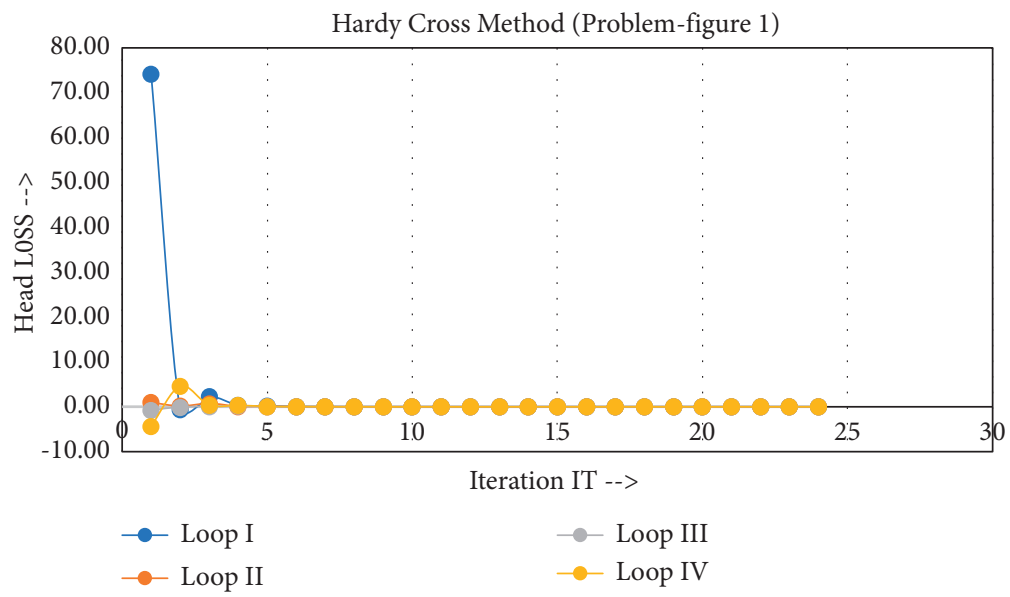

FIGURE 3: The numerical results of head loss of equation (1) by using HCM and head loss in each loop going to zero after $24^{\text {th }}$ iteration by selecting the random original flow in network problem.

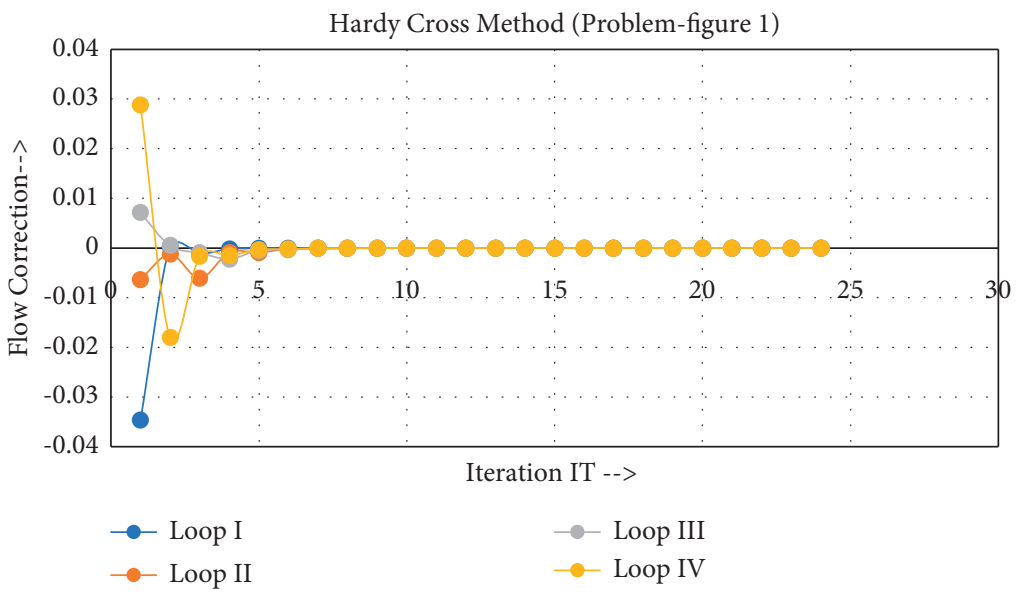

Figure 4: The numerical results of correction flow of equation (1) by using HCM and total corrected flow ( $\mathrm{m}^{3} / \mathrm{s}$ ) in each loop going to nil after $24^{\text {th }}$ iteration with selecting random original flow in network problem, where $\Delta Q_{I}, \Delta Q_{\text {II }}, \Delta Q_{\text {III }}$, and $\Delta Q_{\text {IV }}$ change in flow of loop I-loop IV, respectively.

TABLE 2: The water problem (Figure 2) displaying the first iteration of technique NM ([32]). It is not necessary to follow Kirchhoff's law in NM.

\begin{tabular}{|c|c|c|c|c|c|c|c|c|}
\hline Pipes & Loops & Diameter $(\mathrm{m})$ & Length $(\mathrm{m})$ & $\mathbf{q}\left(\mathrm{m}^{3} / \mathrm{s}\right)$ & Sign $(q)$ & $\mathbf{W}_{\mathbf{w}}$, equation (1) & $\mathrm{NM}, \mathbf{q}\left(\mathrm{m}^{3} / \mathrm{s}\right)$ & $\Delta \mathbf{q}\left(\mathrm{m}^{3} / \mathrm{s}\right)$ \\
\hline 1 & & 0.457 & 120 & 0.125 & 1 & 3.48 & 0.0892094 & -0.0357906 \\
\hline 9 & I & 0.406 & 160 & 0.05 & 1 & 1.34 & 0.0352368 & -0.0147632 \\
\hline 13 & & 0.355 & 120 & -0.025 & -1 & -0.49 & 0.112488 & 0.087488 \\
\hline 7 & & 0.304 & 120 & -0.175 & -1 & -52.34 & 0.210791 & 0.035791 \\
\hline 8 & & 0.304 & 280 & 0.175 & 1 & 122.12 & 0.139209 & -0.035791 \\
\hline & Loop & & & & $\Sigma$ & 74.11 & & 0.0369342 \\
\hline 2 & & 0.457 & 150 & 0.075 & 1 & 1.57 & 0.0539726 & -0.0210274 \\
\hline 10 & II & 0.406 & 160 & 0.04 & 1 & 0.86 & 0.017803 & -0.022197 \\
\hline 11 & & 0.406 & 150 & -0.015 & -1 & -0.11 & 0.0877249 & 0.0727249 \\
\hline 9 & & 0.406 & 160 & -0.05 & -1 & -1.34 & 0.0352368 & -0.0147632 \\
\hline & Loop & & & & $\Sigma$ & 0.97 & & 0.0147373 \\
\hline 3 & & 0.457 & 150 & 0.035 & 1 & 0.34 & 0.0361696 & 0.0011696 \\
\hline 4 & III & 0.406 & 160 & -0.025 & -1 & -0.34 & 0.0238304 & -0.0011696 \\
\hline
\end{tabular}


TABLE 2: Continued.

\begin{tabular}{|c|c|c|c|c|c|c|c|c|}
\hline Pipes & Loops & Diameter (m) & Length (m) & $\mathbf{q}\left(\mathrm{m}^{3} / \mathrm{s}\right)$ & Sign $(q)$ & $\mathbf{W}_{\mathbf{w}}$, equation (1) & $\mathrm{NM}, \mathbf{q}\left(\mathrm{m}^{3} / \mathrm{s}\right)$ & $\Delta \mathbf{q}\left(\mathrm{m}^{3} / \mathrm{s}\right)$ \\
\hline 12 & \multirow{4}{*}{ Loop } & 0.406 & 150 & 0.015 & 1 & 0.11 & -0.0355278 & -0.0505278 \\
\hline 10 & & 0.406 & 160 & -0.04 & -1 & -0.86 & 0.017803 & -0.022197 \\
\hline & & & & & $\Sigma$ & -0.74 & & -0.0727248 \\
\hline 5 & & 0.457 & 120 & -0.09 & -1 & -1.80 & 0.0383025 & -0.0516975 \\
\hline 6 & \multirow[t]{5}{*}{ IV } & 0.609 & 300 & -0.15 & -1 & -2.98 & 0.0983025 & -0.0516975 \\
\hline 13 & & 0.355 & 120 & 0.025 & 1 & 0.49 & 0.112488 & 0.087488 \\
\hline 11 & & 0.406 & 150 & 0.015 & 1 & 0.11 & 0.0877249 & 0.0727249 \\
\hline 12 & & 0.406 & 150 & -0.015 & -1 & -0.11 & -0.0355278 & -0.0505278 \\
\hline & & & & & $\Sigma$ & -4.29 & & 0.0062901 \\
\hline
\end{tabular}

All bold values are based on multidimension.

TABle 3: The first iteration of MNM ([34]) for the water problem (Figure 2).

\begin{tabular}{|c|c|c|c|c|c|c|c|c|}
\hline Pipes & Loops & Diameter $(\mathrm{m})$ & Length (m) & $\mathbf{q}\left(\mathrm{m}^{3} / \mathbf{s}\right)$ & Sign (q) & $\mathbf{W}_{\mathbf{w}}$, equation (1) & MNM, $\mathbf{q}\left(\mathbf{m}^{3} / \mathbf{s}\right)$ & $\Delta \mathbf{q}\left(\mathbf{m}^{3} / \mathbf{s}\right)$ \\
\hline 1 & \multirow{5}{*}{ I } & 0.457 & 120 & 0.125 & 1 & 3.48 & 0.0879253 & -0.0370747 \\
\hline 9 & & 0.406 & 160 & 0.05 & 1 & 1.34 & 0.0315287 & -0.0184713 \\
\hline 13 & & 0.355 & 120 & -0.025 & -1 & -0.49 & 0.115121 & 0.090121 \\
\hline 7 & & 0.304 & 120 & -0.175 & -1 & -52.34 & 0.212075 & 0.037075 \\
\hline 8 & & 0.304 & 280 & 0.175 & 1 & 122.12 & 0.137925 & -0.037075 \\
\hline & \multirow[t]{2}{*}{ Loop } & & & & $\Sigma$ & 74.11 & & 0.034575 \\
\hline 2 & & 0.457 & 150 & 0.075 & 1 & 1.57 & 0.0563965 & -0.0186035 \\
\hline 10 & \multirow[t]{3}{*}{ II } & 0.406 & 160 & 0.04 & 1 & 0.86 & 0.0225996 & -0.0174004 \\
\hline 11 & & 0.406 & 150 & -0.015 & -1 & -0.11 & 0.08665 & 0.07165 \\
\hline 9 & & 0.406 & 160 & -0.05 & -1 & -1.34 & 0.0315287 & -0.0184713 \\
\hline & \multirow[t]{2}{*}{ Loop } & & & & $\Sigma$ & 0.97 & & 0.0171748 \\
\hline 3 & & 0.457 & 150 & 0.035 & 1 & 0.34 & 0.0337969 & -0.0012031 \\
\hline 4 & \multirow[t]{3}{*}{ III } & 0.406 & 160 & -0.025 & -1 & -0.34 & 0.0262031 & 0.0012031 \\
\hline 12 & & 0.406 & 150 & 0.015 & 1 & 0.11 & -0.0392496 & -0.0542496 \\
\hline 10 & & 0.406 & 160 & -0.04 & -1 & -0.86 & 0.0225996 & -0.0174004 \\
\hline & \multirow[t]{2}{*}{ Loop } & & & & $\Sigma$ & -0.74 & & -0.07165 \\
\hline 5 & & 0.457 & 120 & -0.09 & -1 & -1.80 & 0.0369535 & -0.0530465 \\
\hline 6 & \multirow[t]{5}{*}{ IV } & 0.609 & 300 & -0.15 & -1 & -2.98 & 0.0969535 & -0.0530465 \\
\hline 13 & & 0.355 & 120 & 0.025 & 1 & 0.49 & 0.115121 & -0.0134879 \\
\hline 11 & & 0.406 & 150 & 0.015 & 1 & 0.11 & 0.08665 & 0.07165 \\
\hline 12 & & 0.406 & 150 & -0.015 & -1 & -0.11 & -0.0392496 & -0.0542496 \\
\hline & & & & & $\Sigma$ & -4.29 & & -0.1021805 \\
\hline
\end{tabular}

All bold values are based on multidimension.

TABLE 4: The first iteration of method HCM for the water problem (Figure 2).

\begin{tabular}{|c|c|c|c|c|c|c|c|c|c|}
\hline Loops & Pipes & Diameter (m) & Length (m) & $\mathbf{q}\left(\mathrm{m}^{3} / \mathrm{s}\right)$ & Sign (q) & $W_{w}($ equation $(1))$ & $\left|W_{w}^{\prime}\right|$ (equation (2)) & Correction & New $\mathbf{q}\left(\mathbf{m}^{3} / \mathbf{s}\right)$ \\
\hline \multirow[t]{5}{*}{ I } & 1 & 0.457 & 120 & $A_{1}=0.125$ & 1 & 3.48 & 55.65 & & 0.09040843 \\
\hline & 9 & 0.406 & 160 & $A_{2}=0.05$ & 1 & 1.34 & 53.63 & -0.006320742 & 0.021729173 \\
\hline & 13 & 0.355 & 120 & $A_{3}=-0.025$ & -1 & -0.49 & 39.35 & 0.028735632 & -0.088327201 \\
\hline & 7 & 0.304 & 120 & $A_{4}=-0.175$ & -1 & -52.34 & 598.13 & & -0.20959157 \\
\hline & 8 & 0.304 & 280 & $A_{5}=0.175$ & 1 & 122.12 & 1395.65 & & 0.14040843 \\
\hline \multirow[t]{3}{*}{ Loop } & & & & & $\Sigma$ & 74.11 & 2142.40 & & \\
\hline & & & & & & $-\mathbf{W}_{\mathbf{w}} /\left|\mathbf{W}_{\mathbf{w}}^{\prime}\right|$ & -0.03459157 & & \\
\hline & 2 & 0.457 & 150 & $B_{1}=0.075$ & 1 & 1.57 & 41.73 & & 0.068679258 \\
\hline \multirow[t]{3}{*}{ II } & 10 & 0.406 & 160 & $B_{2}=0.04$ & 1 & 0.86 & 42.90 & 0.007089585 & 0.026589673 \\
\hline & 11 & 0.406 & 150 & $B_{3}=-0.015$ & -1 & -0.11 & 15.08 & 0.028735632 & -0.050056374 \\
\hline & 9 & 0.406 & 160 & $B_{4}=-0.05$ & -1 & -1.34 & 53.63 & -0.03459157 & -0.021729173 \\
\hline \multirow[t]{3}{*}{ Loop } & & & & & $\Sigma$ & 0.97 & 153.35 & & \\
\hline & & & & & & $-\mathbf{W}_{\mathbf{w}} /\left|\mathbf{W}_{\mathbf{w}}^{\prime}\right|$ & -0.006320742 & & \\
\hline & 3 & 0.457 & 150 & $C_{1}=0.035$ & 1 & 0.34 & 19.48 & & 0.042089585 \\
\hline III & 4 & 0.406 & 160 & $C_{2}=-0.025$ & -1 & -0.34 & 26.81 & & -0.017910415 \\
\hline
\end{tabular}


TABLE 4: Continued.

\begin{tabular}{|c|c|c|c|c|c|c|c|c|c|}
\hline Loops & Pipes & Diameter $(\mathrm{m})$ & Length $(\mathrm{m})$ & $\mathbf{q}\left(\mathrm{m}^{3} / \mathrm{s}\right)$ & Sign (q) & $W_{w}($ equation $(1))$ & $\left|W_{w}^{\prime}\right|$ (equation (2)) & Correction & New $\mathbf{q}\left(\mathbf{m}^{3} / \mathbf{s}\right)$ \\
\hline \multirow{5}{*}{ Loop } & 12 & 0.406 & 150 & $C_{3}=0.015$ & 1 & 0.11 & 15.08 & 0.028735632 & -0.006646047 \\
\hline & 10 & 0.406 & 160 & $C_{4}=-0.04$ & -1 & -0.86 & 42.90 & -0.006320742 & -0.026589673 \\
\hline & & & & & $\Sigma$ & -0.74 & 104.27 & & \\
\hline & & & & & & $-\mathbf{W}_{\mathbf{w}} /\left|\mathbf{W}_{\mathbf{w}}^{\prime}\right|$ & 0.007089585 & & \\
\hline & 5 & 0.457 & 120 & -0.09 & -1 & -1.80 & 40.06 & & -0.061264368 \\
\hline \multirow[t]{6}{*}{ IV } & 6 & 0.609 & 300 & -0.15 & -1 & -2.98 & 39.72 & & -0.121264368 \\
\hline & 13 & 0.355 & 120 & 0.025 & 1 & 0.49 & 39.35 & -0.03459157 & 0.088327201 \\
\hline & 11 & 0.406 & 150 & 0.015 & 1 & 0.11 & 15.08 & -0.006320742 & 0.050056374 \\
\hline & 12 & 0.406 & 150 & -0.015 & -1 & -0.11 & 15.08 & 0.007089585 & 0.006646047 \\
\hline & & & & & $\Sigma$ & -4.29 & 149.30 & & \\
\hline & & & & & & $-\mathbf{W}_{\mathbf{w}} /\left|\mathbf{W}_{\mathbf{w}}^{\prime}\right|$ & 0.028735632 & & \\
\hline
\end{tabular}

All bold values are based on multidimension.

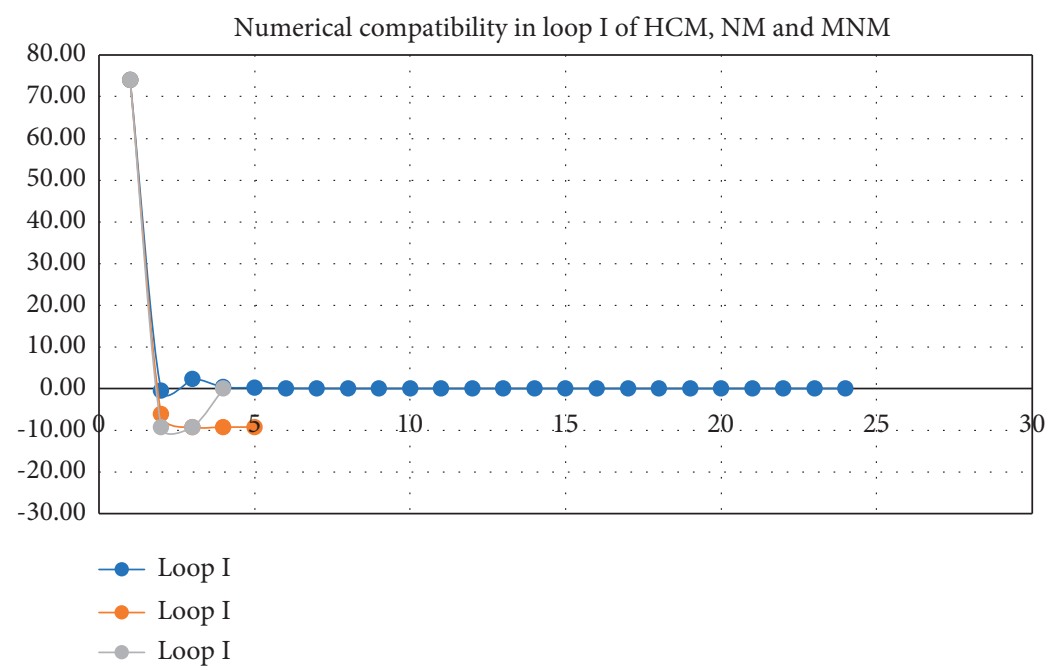

FIGURE 5: The numerical compatibility of all HCM, NM, and MNM approaches in loop I.

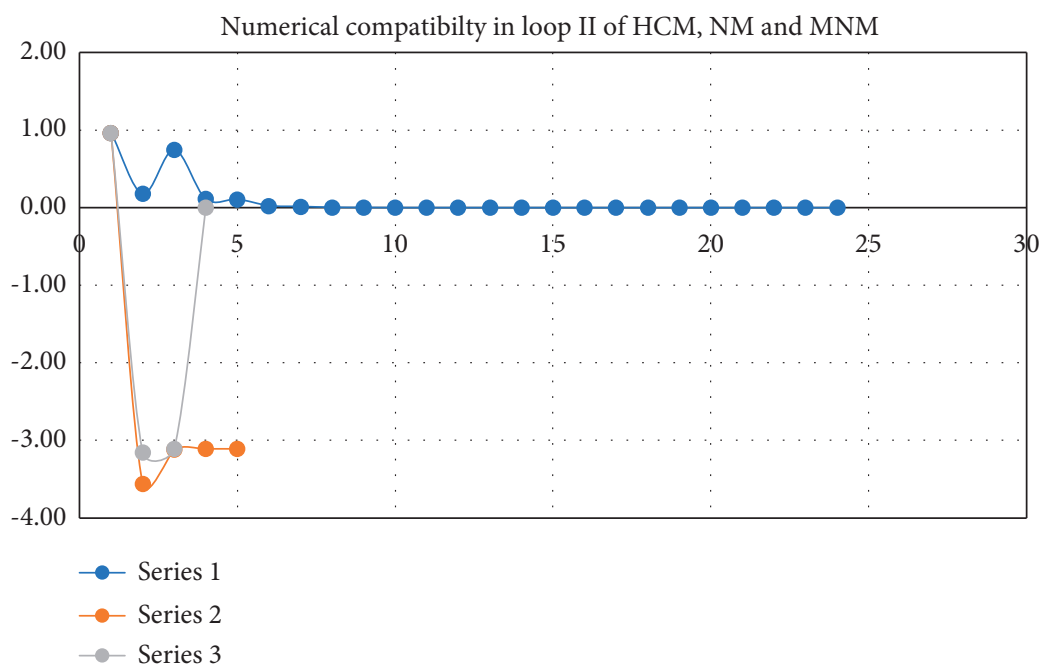

Figure 6: The numerical compatibility of all HCM, NM, and MNM approaches in loop II. 


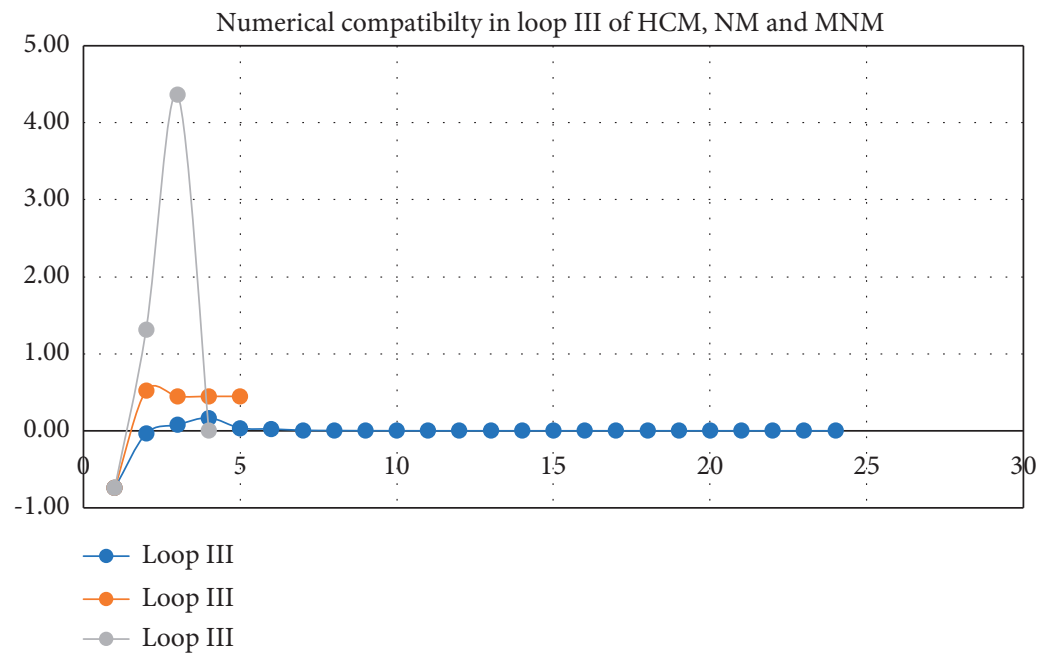

FIgure 7: The numerical compatibility of all HCM, NM, and MNM approaches in loop III.

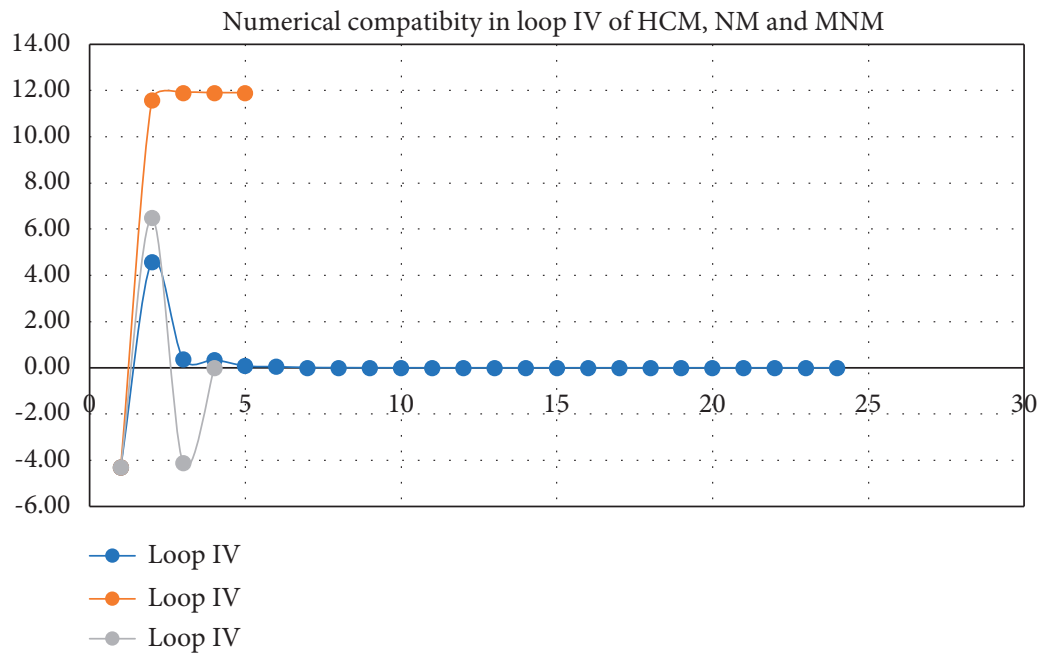

FIGURE 8: The numerical compatibility of all HCM, NM, and MNM approaches in loop IV.

Table 5

\begin{tabular}{|c|c|c|c|c|c|}
\hline Start node & End node & Pipe index & Hardy Cross method $\left(\mathrm{m}^{3} / \mathrm{s}\right)$ & Newton method $\left(\mathrm{m}^{3} / \mathrm{s}\right)$ & Modified NM $\left(\mathrm{m}^{3} / \mathrm{s}\right)$ \\
\hline $\bar{A}$ & B & 1 & 0.0860912 & 0.0860912 & 0.0860912 \\
\hline B & $\mathrm{C}$ & 2 & 0.057979 & 0.057979 & 0.057979 \\
\hline $\mathrm{C}$ & $\mathrm{D}$ & 3 & 0.0380804 & 0.0380804 & 0.0380804 \\
\hline $\mathrm{D}$ & $\mathrm{G}$ & 4 & (a) -0.0219196 & 0.0219196 & 0.0219196 \\
\hline G & $\mathrm{J}$ & 5 & -0.0854642 & 0.0854642 & 0.0854642 \\
\hline $\mathrm{J}$ & I & 6 & -0.145464 & 0.145464 & 0.145464 \\
\hline I & $\mathrm{H}$ & 7 & -0.213909 & 0.213909 & 0.213909 \\
\hline $\mathrm{H}$ & $\mathrm{A}$ & 8 & 0.136091 & 0.136091 & 0.136091 \\
\hline B & $\mathrm{E}$ & 9 & 0.0281122 & 0.0281122 & 0.0281122 \\
\hline $\mathrm{C}$ & $\mathrm{F}$ & 10 & -0.0198986 & 0.0198986 & 0.0198986 \\
\hline $\mathrm{E}$ & $\mathrm{F}$ & 11 & 0.0365569 & Hoboken & 0.0365569 \\
\hline $\mathrm{F}$ & G & 12 & -0.0135446 & 0.0135446 & 0.0135446 \\
\hline $\mathrm{E}$ & I & 13 & 0.0684447 & 0.0684447 & 0.0684447 \\
\hline
\end{tabular}

${ }^{(a)}$ The stream is moving in the opposite direction of the original flow, as indicated by the negative signs. The final water flow of HCM, NM, and MNM is explained in columns 7-9. 


\section{Analysis and Results Discussion}

Several processes must be carried out in order for this to operate. The recommended effort is run on a 10th Generation Intel Core i7 processor, 1 TB SSD, and GTX $1660 \mathrm{Ti}$ (6 GB) graphics card with Windows 10 as the operating system. We used Mathematica 11.2 to do all of the existing simulations. The statistics in Table 4 show only the first iteration of HCM, NM, and MNM, respectively. These results show that HCM reaches its ideal level after 24 rounds, NM after 5 iterations, and MNM after 3 iterations.

Figures 5-8 show the evaluation and assessment of HCM, NM, and MNM in each loop, demonstrating that MNM is less expensive and takes less time to converge than traditional HCM and NM approaches. Table 5 provides the findings and evaluation of head loss in each loop of all approaches.

The optimality status of HCM, NM, and MNM is given in Table 5. We may also check that the original scheme, when compared to HCM and NM, achieves an optimal level with fewer steps and less time. On the other hand, HCM reaches the optimal stage after 24 rounds, NM after 5 iterations, and MNM after 3 iterations. The MNM's key benefit is this statistic. This study's novelty and effectiveness can be seen in these results.

\section{Conclusion}

The friction factor $f$ is calculated using (3) in the first stage of this study. In a retraction domain $\operatorname{Re}>4000$, this equation represents the relationship between the tube's innermost diameter, tube roughness, fraction factor, and Reynolds number. This relationship has an implied form that is impossible to solve explicitly. To circumvent this limitation, we numerically solved (3) using the most recent and up-to-date modified Chun-Hui He's Algorithm [24] based on the sizes of each pipe in the system, as shown in Figure 2. The numerical value of the fraction factor was then used to calculate the water pressure function, which was then utilized to estimate the head loss and corrected flow between two consecutive values using the Hardy Cross method, Newton method, and modified Newton method. Tables 2-4 provide that HCM obtains the needed solution after 24 iterations, NM after 5 iterations, and MNM after 3 iterations, all with stopping criteria $1 \times 10^{-10}$.

\section{Nomenclature}

HCM: Hardy Cross method

$\pi: \quad$ Ludolph's number $(=3.14159)$

$f: \quad$ Friction function

$l$ : $\quad$ Length $(\mathrm{m} / \mathrm{s})$

$\varepsilon: \quad$ Roughness height

$q$ : $\quad$ Discharge pressure (1/s)

PVC: Polyvinyl chloride

d: $\quad$ Diameter (m)

BVPs: Boundary value problems

MNM: Modified Newton method

$g: \quad 9.8\left(\mathrm{~m} / \mathrm{s}^{2}\right)$ $f: \quad$ Friction factor

NM: Newton method

Re: Reynolds number

$G_{g}:$ : Head loss

IT: Iteration.

\section{Data Availability}

The data used to support the findings of this study are available from the corresponding author upon request.

\section{Conflicts of Interest}

The authors declare that they have no conflicts of interest.

\section{Authors' Contributions}

W.A. and M.A. developed methodology and wrote and reviewed the article. W.A. investigated the study and wrote the original draft. W.A., A.J.R., and U.F. collected resources. Z.A. and F.F. collected data. M.A. conceptualized the study. W.A. and J.R. developed software. M.K. performed formal analysis.

\section{Acknowledgments}

The authors extend their appreciation to the deputyship for Research and Innovation, Ministry of Education in Saudi Arabia for funding this research work (IFP-2020-20).

\section{References}

[1] J. H. He, "Ancient Chinese Algorithm: the Ying Buzu Shu (method of surplus and deficiency) vs. Newton iteration method," Journal of Applied Mathematics and Mechanics, vol. 23, no. 12, pp. 1407-1412, 2002, English Edition.

[2] J.-H. He, "Solution of nonlinear equations by an ancient Chinese algorithm," Applied Mathematics and Computation, vol. 151, no. 1, pp. 293-297, 2004.

[3] W. A. Khan, "Numerical and simulation analysis comparison of hydraulic network problem base on higher-order efficiency approach," Alexandria Engineering Journal, vol. 60, no. 5, pp. 4889-4903, 2021.

[4] M. Bagajewicz and G. Valtinson, "Computation of natural gas pipeline hydraulics," Industrial \& Engineering Chemistry Research, vol. 53, no. 26, pp. 10707-10720, 2014.

[5] D. Brkić and P. Praks, "An efficient iterative method for looped pipe network hydraulics free of flow-corrections," Fluid, vol. 4, pp. 73 1-19, 2019.

[6] D. Brkić, "Solution of the implicit Colebrook equation for flow friction using Excel sheets Educ," vol. 10, no. 2, 2019, https:// sie.scholasticahq.com/article/4663-solution-of-the-implicitcolebrook-equation-for-flow $\% 20$ friction-using-excel.

[7] A. Cordero, E. Martínez, and J. R. Torregrosa, "Iterative methods of order four and five for systems of nonlinear equations," Journal of Computational and Applied Mathematics, vol. 231, no. 2, pp. 541-551, 2009.

[8] D. Brki'c and P. Praks, "Accurate and efficient explicit approximations of the Colebrook flow friction equation based on the wright $\omega$-function," Mathematics, vol. 7, no. 34, 2019.

[9] D. Brkić and P. Praks, "Short overview of early developments of the Hardy cross type methods for computation of flow 
distribution in pipe networks," Applied Sciences, vol. 9, no. 10, 2019.

[10] O. Bello, A. Abu-Mahfouz, Y. Hamam, P. R. Page, K. B. Adedeji, and O. Piller, "Solving management problems in water distribution networks: a survey of approaches and mathematical models," Water, vol. 11, no. 3, p. 562, 2019.

[11] P. Carpentier and G. Cohen, "Applied mathematics in water supply network management," Automatica, vol. 29, no. 5, pp. 1215-1250, 1993.

[12] J. H. He and Y. O. El-Dib, "The reducing rank method to solve third-order Duffing equation with the homotropy perturbation," Numerical Methods for Partial Differential Equations, vol. 37, no. 4, pp. 1-9, 2020.

[13] O. Abu Arqub, "Numerical solutions of systems of first-order, two-point BVPs based on the reproducing kernel algorithm," Calcolo, vol. 55, no. 3, p. 31, 2018.

[14] G. Cembrano, G. Wells, J. Quevedo, R. Pérez, and R. Argelaguet, "Optimal control of a water Distribution network in a supervisory control system," Control Engineering Practice, vol. 8, no. 10, pp. 1177-1188, 2000.

[15] H. Cross, "Analysis of Flow in Networks of Conduits or Conductors," Bulletin No. 286, University of Illinois Engineering Experimental Station, Urbana, III., 1936.

[16] E. Ifiemi, J. Sodiki, and B. Nkoi, "Analysis of a water distribution network by Newton-raphson multivariable method: a case of negligible minor losses," Innovative Systems Design and Engineering, vol. 11, no. 2, pp. 15-28, 2020.

[17] P. K. Swamee and A. K. Sharma, Design of Water Supply Pipe Networks, John Wiley \& Sons, Hoboken, NJ, USA, 2008.

[18] R. Epp and A. G. Fowler, "Efficient code for steady-state flows in networks," Journal of Hydraulics Division, ASCE, vol. 96, no. HY1, pp. 43-56, 1970.

[19] H. B. Nielsen, "Methods for analyzing pipe networks," ASCE Journals of Hydraulic Engineering, vol. 115, no. 2, pp. 139-157, 1990.

[20] E. Toldini and S. Pilati, "A gradient algorithm for the analysis of pipe networks," International Conference on Computer Applications for Water Supply and Distribution (System Analysis and Simulation), vol. 1, pp. 1-20, John Wiley \& Sons, London, UK, 1988.

[21] H. A. Basha and B. G. Kassab, "Analysis of water distribution systems using a perturbation method," Applied Mathematical Modelling, vol. 20, no. 4, pp. 290-297, 1996.

[22] C.-H. He, "An introduction to an ancient Chinese algorithm and its modification," International Journal of Numerical Methods for Heat and Fluid Flow, vol. 26, no. 8, pp. 24862491, 2016.

[23] H. S. Abdul, "Design of nonlinear piping water system network by using Newton raphson method," Journal of Mechanical and Civil Engineering (IOSR-JMCE), vol. 13, no. 2, pp. 33-39, 2016.

[24] W. A. Khan, "Numerical simulation of Chun-Hui He's iteration method with applications in engineering," International Journal of Numerical Methods for Heat and Fluid Flow, vol. 32, no. 3, pp. 944-955, 2022.

[25] P. Praks and D. Brkić, "Choosing the optimal multi-point iterative method for the Colebrook flow friction equation," Processes, vol. 6, no. 8, p. 130, 2018.

[26] C. F. Colebrook, "Turbulent flow in pipes, with particular reference to the transition region between the smooth and rough pipe laws," Journal of the Institution of Civil Engineers, vol. 11, no. 4, pp. 133-156, 1939.
[27] B. Gay and P. Middleton, "The solution of pipe network problems," Chemical Engineering Science, vol. 26, no. 1, pp. 109-123, 1971.

[28] D. Brki'c and Ž. Cojbaši 'c, "Evolutionary optimization of Colebrook's turbulent flow friction approximations," Fluid, vol. 2, no. 15, 2017.

[29] D. W. Martins and G. Peters, "The application of Newton's method to network analysis by digital computer," Journal of the Institution of Water Engineering, vol. 17, pp. 115-129, 1963.

[30] M. H. Afshar and A. Afshar, "An element-by-element algorithm for pipe network analysis," Transactions on Ecology and the Environment, vol. 30, pp. 1743-3541, 1999.

[31] D. Brki'c, "An Improvement of Hardy Cross method applied on looped spatial natural gas distribution networks," Applied Energy, vol. 86, pp. 1290-1300, 2009.

[32] J. F. Traub, Iterative Methods for the Solution of Equations, Chelsea Publishing Company, New York, NY, USA, 1982.

[33] N. Moosavian and M. R. Jaefarzadeh, "Hydraulic Analysis of water supply networks using a modified hard Darcy method," International Journal of Engineering, vol. 27, no. 9, pp. 1331-1338, 2014.

[34] W. A. Khan, K. I. Noor, K. Bhatti, and F. A. Ansari, “A new fourth order Newton-type method for solution of system of nonlinear equations," Applied Mathematics and Computation, vol. 270, pp. 724-730, 2015. 